\title{
Mott transition in two-dimensional frustrated compounds
}

\author{
A. Liebsch, ${ }^{1}$ H. Ishida, ${ }^{2}$ and J. Merino ${ }^{3}$ \\ ${ }^{1}$ Institut für Festkörperforschung, Forschungszentrum Jülich, 52425 Jülich, Germany \\ ${ }^{2}$ College of Humanities and Sciences, Nihon University, Tokyo 156, Japan \\ ${ }^{3}$ Departamento de Física Teórica de la Materia Condensada, Universidad Autónoma de Madrid, Madrid 28049, Spain
}

(Received 12 March 2009; published 11 May 2009)

\begin{abstract}
The phase diagrams of isotropic and anisotropic triangular lattices with local Coulomb interactions are evaluated within cluster dynamical mean-field theory. As a result of partial geometric frustration in the anisotropic lattice, short-range correlations are shown to give rise to re-entrant behavior which is absent in the fully frustrated isotropic limit. The qualitative features of the phase diagrams including the critical temperatures are in good agreement with experimental data for the layered organic charge-transfer salts $\kappa-(\mathrm{BEDT}-\mathrm{TTF})_{2} \mathrm{Cu}\left[\mathrm{N}(\mathrm{CN})_{2}\right] \mathrm{Cl}$ and $\kappa$-(BEDT-TTF $)_{2} \mathrm{Cu}_{2}(\mathrm{CN})_{3}$.
\end{abstract}

DOI: 10.1103/PhysRevB.79.195108

PACS number(s): 71.10.Fd

\section{INTRODUCTION}

The influence of spatial quantum fluctuations on the nature of the Mott transition in strongly correlated systems is currently of great interest. A class of materials in which these effects can be studied in detail are the layered charge-transfer salts of the $\kappa$-(BEDT-TTF $)_{2} X$ family, where $X$ denotes an inorganic monovalent anion such as $\mathrm{Cu}\left[\mathrm{N}(\mathrm{CN})_{2}\right] \mathrm{Cl}$ or $\mathrm{Cu}_{2}(\mathrm{CN})_{3}$. The electronic properties of these compounds have been shown to be highly sensitive functions of hydrostatic pressure. ${ }^{1-8}$ As a result, the temperature versus pressure phase diagram is remarkably rich, exhibiting Fermiliquid and bad-metallic behavior, superconductivity as well as paramagnetic and antiferromagnetic (AF) insulating phases. These observations suggest fascinating connections to analogous phenomena in various transition-metal oxides. ${ }^{9}$

A feature of particular interest in the organic salts is magnetic frustration. Since the geometric structure corresponds to an anisotropic triangular lattice with inequivalent nearest-neighbor hopping interactions $t$ and $t^{\prime},{ }^{10,11}$ longrange magnetic ordering becomes increasingly frustrated if the lattice is nearly isotropic, giving rise to an exotic spinliquid phase in the absence of symmetry breaking. ${ }^{12}$ Such a spin-liquid phase ${ }^{5,7,13}$ is realized in the organic insulator $\kappa$-(BEDT-TTF $)_{2} \mathrm{Cu}_{2}(\mathrm{CN})_{3}$ (denoted below as $\kappa$-CN) which corresponds to $t^{\prime} \approx 1.06 t$, whereas $\kappa$-(BEDT-TTF) ${ }_{2} \mathrm{Cu}\left[\mathrm{N}(\mathrm{CN})_{2}\right] \mathrm{Cl}$ (denoted as $\kappa$-Cl) with $t^{\prime}$ $\approx 0.75 t$ is an $\mathrm{AF}$ insulator. ${ }^{3,4} \mathrm{AF}$ order is also found in those $\operatorname{Pd}(\operatorname{dim} t)_{2}$ salts for which $0.55<t^{\prime} / t<0.85$. In contrast, $\mathrm{C}_{2} \mathrm{H}_{5}\left(\mathrm{CH}_{3}\right)_{3} \mathrm{P}\left[\mathrm{Pd}(\mathrm{dimt})_{2}\right]_{2}$ with $t^{\prime}=1.05 t$ is a valence-bond solid insulator at ambient pressure. ${ }^{14}$ Experiments on these kinds of two-dimensional frustrated systems have greatly stimulated theoretical investigations of the electronic and magnetic properties of triangular lattices. ${ }^{15-28}$

The focus of the present study is the bandwidth-controlled finite-temperature phase diagram of the Hubbard model for isotropic and anisotropic triangular lattices. The key result is that small changes in the ratio $t^{\prime} / t$ can give rise to fundamental changes of the phase diagram. Thus, partial and full magnetic frustration reveal strikingly different metalinsulator coexistence regions in qualitative agreement with the experimental phase diagrams for $\kappa$-Cl (Ref. 4) and $\kappa$-CN. ${ }^{7}$
The anisotropic triangular lattice has recently been studied also by Ohashi et al. $^{26}$ who used dynamical mean-field theory (DMFT) (Ref. 29) with a cluster extension to account for spatial fluctuations. Although at $t^{\prime} \approx 0.8 t$ re-entrant behavior was found as observed for $\kappa$ - $\mathrm{Cl}$, the calculated $T_{c}$ was much larger than the measured value. Moreover, only the lower boundary of the metal-insulator coexistence region was determined. Here, we investigate both the isotropic and anisotropic triangular lattices and use exact diagonalization (ED) (Ref. 30) combined with cluster DMFT (Ref. 31) to evaluate the upper and lower phase boundaries, $U_{c 1}(T)$ and $U_{c 2}(T)$, of the coexistence region. As shown below, the shape of these boundaries as well as the critical temperatures are consistent with the experimental data for $\kappa-\mathrm{Cl}$ and $\kappa$ - CN.

\section{THEORY AND RESULTS}

The minimal model Hamiltonian that captures the interplay between geometrical frustration and strong Coulomb interaction present in the conducting layers of organic salts such as $\kappa-\mathrm{Cl}$ and $\kappa-\mathrm{CN}$ is

$$
H=-\sum_{i j \sigma} t_{i j}\left(c_{i \sigma}^{+} c_{j \sigma}+\text { H.c. }\right)+U \sum_{i} n_{i \uparrow} n_{i \downarrow}-\mu \sum_{i \sigma} c_{i \sigma}^{+} c_{i \sigma},
$$

where the sum in the first term is limited to nearest-neighbor sites. The hopping integrals in a unit cell consisting of three sites are $t_{13}=t_{23}=t$ and $t_{12}=t^{\prime}$. The bandwidth is $W=9 t$ for $t^{\prime}=t$ and $W=8.5 t$ for $t^{\prime}=0.8 t$. The chemical potential $\mu$ is fixed to give half filling. Within cluster DMFT the interacting lattice Green's function in the cluster site basis is defined as

$$
G_{i j}\left(i \omega_{n}\right)=\sum_{\vec{k}}\left[i \omega_{n}+\mu-t(\vec{k})-\Sigma\left(i \omega_{n}\right)\right]_{i j}^{-1},
$$

where $\vec{k}$ extends over the reduced Brillouin zone and $\omega_{n}$ $=(2 n+1) \pi T$ are the Matsubara frequencies. $t(\vec{k})$ denotes the hopping matrix for the superlattice and $\Sigma\left(i \omega_{n}\right)$ represents the nondiagonal cluster self-energy matrix. This self energy is calculated within ED where the environment of the three-site cluster is replaced via a bath consisting of six or nine levels, i.e., for a total cluster size $n_{s}=9$ or $n_{s}=12$. The calculations are carried out on a site basis and on a mixed site/molecular- 

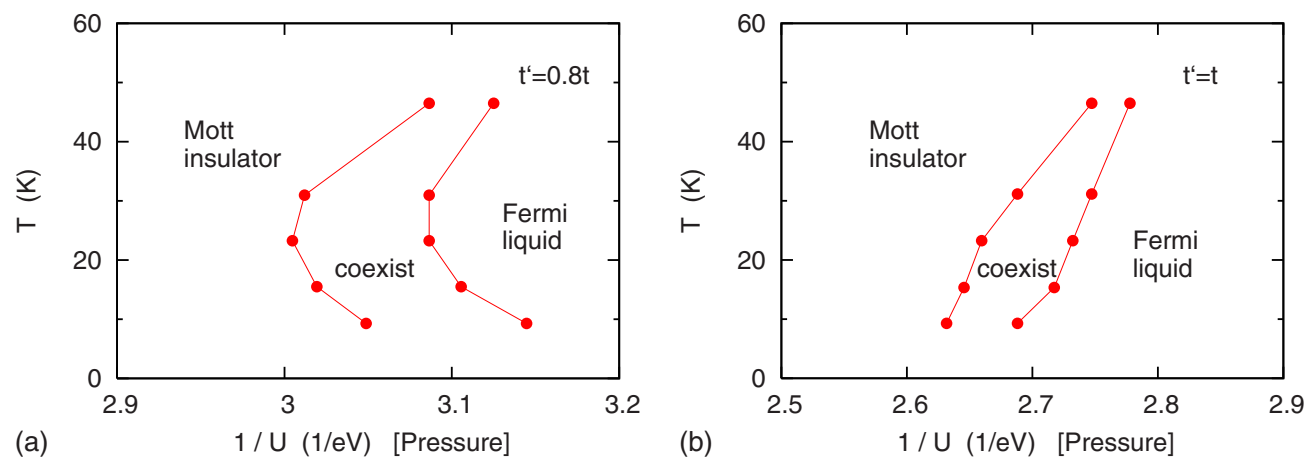

FIG. 1. (Color online) Phase diagrams of Hubbard model for anisotropic triangular lattice $\left(t^{\prime}=0.8 t\right)$ and isotropic triangular lattice $\left(t^{\prime}=t\right)$, evaluated within ED cluster DMFT for $t=0.04 \mathrm{eV}$. Plotted are the first-order metal-insulator phase boundaries as functions of inverse local Coulomb energy $U$. In the experimental setup increasing hydrostatic pressure $P$ implies increasing bandwidth $W$ or decreasing $U$. The re-entrant behavior found for $t^{\prime}=0.8 t$ is absent in the isotropic limit $t^{\prime}=t$.

orbital basis. Due to ED finite-size effects, these treatments give results that differ quantitatively. Nevertheless, the qualitative features of the phase diagrams are consistently reproduced by these ED versions. Details of the cluster ED/DMFT formalism can be found in Ref. 32 .

Figure 1(a) shows the calculated phase diagram for the anisotropic lattice in the region below the critical temperature. To facilitate the comparison with the experimental data for $\kappa-\mathrm{Cl},{ }^{4}$ the hopping matrix elements are chosen as $t$ $=0.04 \mathrm{eV}$ and $t^{\prime}=0.8 t$ to reproduce the single-particle bandwidth, $W=0.34 \mathrm{eV} .{ }^{33}$ A similar value was used in the numerical renormalization group (NRG) DMFT analysis of the high- $T$ data in Ref. 4. Since the data were plotted in a $T / P$ phase diagram, we show the transition temperatures as functions of the inverse Coulomb energy. Increasing pressure $P$ implies increasing electronic bandwidth so that this measurement is equivalent to keeping $W$ fixed and reducing $U$ in the calculation. The phase boundaries of the coexistence region are obtained by carefully increasing or decreasing $U$ from the metallic or insulating domains, respectively. Figure 1(b) shows the phase diagram for the isotropic case corresponding to $\kappa$-CN.

The critical temperatures for $t^{\prime}=0.8 t$ and $t^{\prime}=t, T_{c}$ $\approx 50 \mathrm{~K} \approx 0.11 t$, are consistent with the measured values $T_{c}$ $\approx 40 \mathrm{~K}$ for $\kappa-\mathrm{Cl}$ (Ref. 4) and $T_{c} \approx 50 \mathrm{~K}$ for $\kappa$-CN. ${ }^{7} T_{c}$ $\approx 0.1 t$ was recently obtained also for the fully unfrustrated square lattice. ${ }^{34}$ On the other hand, within quantum Monte Carlo (QMC) DMFT at temperatures $T=0.1 t-1.0 t$, Ohashi $e t$ $a l .{ }^{26}$ found a much larger value $T_{c} \approx 0.3 t \approx 140 \mathrm{~K}$. The experimental data and the present ED/DMFT results suggest that the metal-insulator coexistence region is located at temperatures below those considered in Ref. 26.

For $t^{\prime}=0.8 t$, the first-order phase boundaries separating the Fermi liquid from the Mott insulator in Fig. 1(a) show the same kind of re-entrant behavior as measured for $\kappa$-Cl. For instance, at $U=\frac{1}{3} \mathrm{eV}$ and $T \approx 50 \mathrm{~K}$ the system is a Mott insulator which turns into a Fermi liquid if $T$ is lowered to about $20 \mathrm{~K}$. Further reduction in $T$ reverts the system to a Mott insulator, just as seen in the data (we do not consider here the antiferromagnetic insulating phase which is detected at even lower temperature). Ohashi et al. ${ }^{26}$ found re-entrant behavior at considerably higher temperatures.
At present the origin of differences between the phase diagram for $t^{\prime}=0.8 t$ shown in Fig. 1 and the one found by Ohashi et al. is not clear. One reason might be that we consider a triangular lattice (three sites per cluster) while in Ref. 26 a square lattice with one diagonal was used (four sites per cluster). However, since the experimentally observed critical temperature is much lower than the range treated in Ref. 26, it would be interesting to apply continuous-time QMC to this problem in order to reach lower temperatures.

The re-entrant behavior for $t^{\prime}=0.8 t$ is in striking contrast to the phase diagram obtained for the isotropic triangular lattice shown in Fig. 1(b). This limit resembles more closely the phase diagram derived within single-site DMFT. ${ }^{29}$ The main effect of short-range fluctuations in the isotropic case is a significant lowering of the critical Coulomb energy. Here, $U_{c 2} \approx 1 / 2.63 \mathrm{eV} \approx 9.5 t$, whereas $U_{c 2} \approx 12 t-15 t$ in local DMFT for the triangular lattice. ${ }^{35,36}$ Comparing Figs. 1(a) and 1(b), it is evident that anisotropy causes a further lowering of the critical Coulomb energies. This trend is consistent with $U_{c} \approx 6 t$ for the fully unfrustrated square lattice ${ }^{34,37}$ which is topologically equivalent to the triangular lattice in the limit $t^{\prime}=0$.

It is interesting also to analyze the width of the metalinsulator coexistence region obtained by increasing vs decreasing pressure. For $\kappa-\mathrm{Cl}$, it is observed at $P$ $\approx 200-400$ bar, which according to the high- $T$ NRG analy-

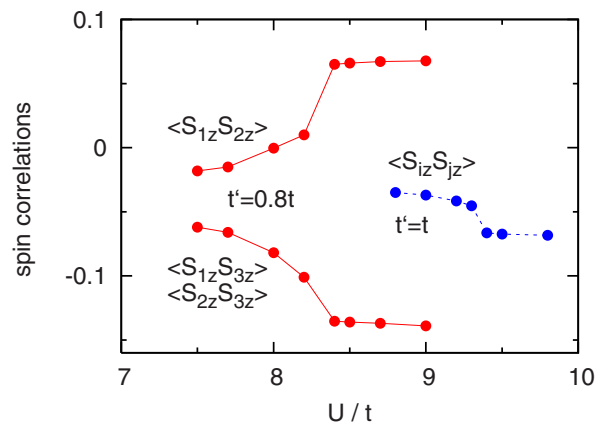

FIG. 2. (Color online) Nearest-neighbor spin correlations in isotropic and anisotropic triangular lattices for $T=0.05 t, t^{\prime}=t_{12}$, and $t=t_{13}=t_{23}$. Strong enhancement of spin correlations occurs for moderate deviations from the isotropic limit. 

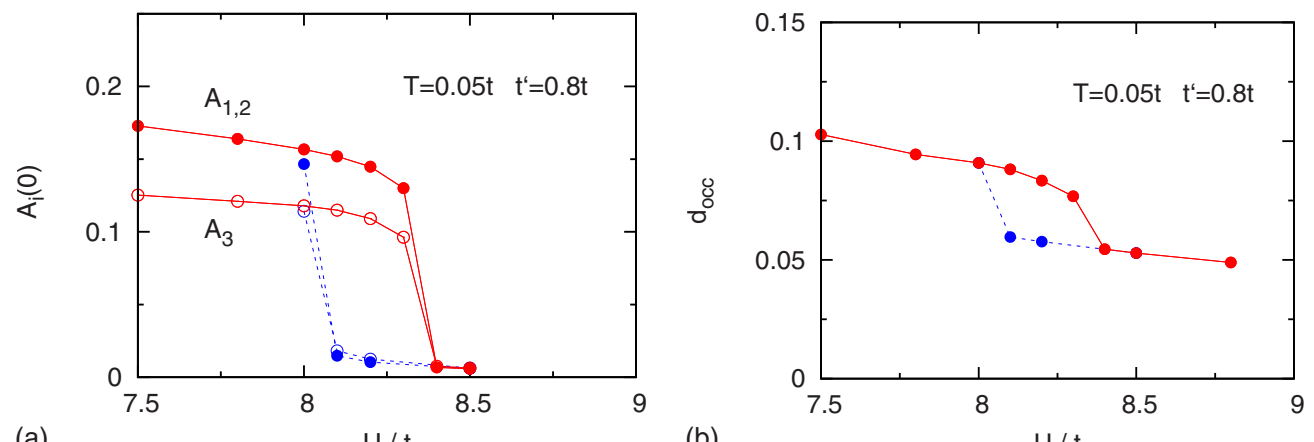

(a)

$\mathrm{U} / \mathrm{t}$

(b)

$\mathrm{U} / \mathrm{t}$

FIG. 3. (Color online) Hysteresis behavior of spectral weights $A_{i}(0)$ at $E_{F}=0$ and average double occupancy $d_{\text {occ }}$ as functions of Coulomb energy for anisotropic triangular lattice $\left(t=1, t^{\prime}=0.8 t\right.$, and $\left.T=0.05 t\right)$. Red solid (blue dashed) curves: increasing (decreasing) $U$.

sis corresponds to bandwidth changes of about $2 \% .^{4}$ The calculated coexistence region shown in Fig. 1(a) is only slightly wider than this experimental range.

The qualitative change in the phase diagram caused by reduced geometrical frustration can be understood by analyzing the magnetic properties of the frustrated lattice. In the $U \rightarrow \infty$ limit, the Hubbard model can be mapped onto the anisotropic Heisenberg model with $J^{\prime} / J=0.64$ and $J^{\prime} / J=1$ for $t^{\prime}=0.8 t$ and $t^{\prime}=t$, respectively. At $T=0, t^{\prime}=t$ yields longrange $\mathrm{AF}$ order of the $120^{\circ}$ type whereas $t^{\prime}=0.8 t$ gives rise to row-wise AF Néel order. ${ }^{38}$ However, in the Heisenberg model the temperature scale for AF order in the isotropic triangular lattice is expected to be strongly suppressed relative to the square lattice. For the Hubbard model, the cluster DMFT provides information on how the magnetic correlations $\left\langle S_{i z} S_{j z}\right\rangle$ vary across the Mott transition in the isotropic case compared with $t^{\prime}=0.8 t$. The results shown in Fig. 2 demonstrate that spin correlations are strongly enhanced as the geometrical frustration is suppressed. The isotropic lattice displays weak AF coupling for any $U$. This is in contrast to $t^{\prime}=0.8 t$, for which the weaker hopping amplitude displays ferromagnetic correlations whereas spins with the larger hopping amplitude are antiferromagnetically coupled, indicating a row-wise AF Neel arrangement of spins. Thus, $t^{\prime}=0.8 t$ induces a much stronger tendency toward magnetic order than $t^{\prime}=t$, which explains why the re-entrant behavior occurs for $t^{\prime}=0.8 t$ but not for $t^{\prime}=t$ (see Fig. 1). At low $T$, the electron entropy is suppressed for $t^{\prime}=0.8 t$ as compared to $t^{\prime}=t$. As $T$ is increased for $t^{\prime}=0.8 t$, the system lowers its free energy by transforming to a metal since the entropy of the

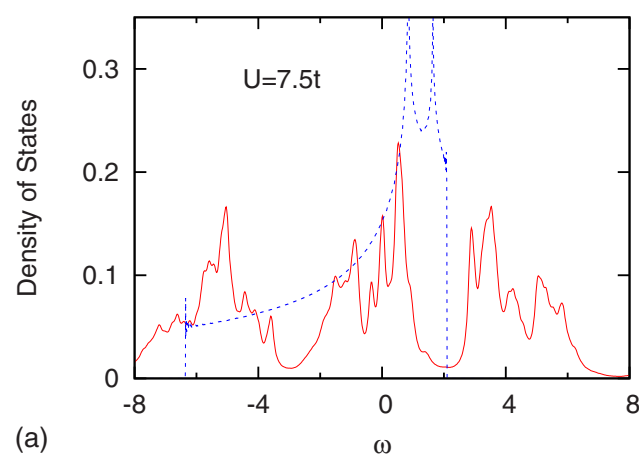

(a) metal exceeds that of the ordered insulator. At even higher temperatures the system gains entropy of log (2) by transforming back into a paramagnetic insulator. This result is analogous to the one found for the unfrustrated square lattice. ${ }^{34}$ In the isotropic lattice magnetic ordering is suppressed and the re-entrant behavior disappears.

To illustrate the first-order nature of the metal-insulator transition we show in the left panel of Fig. 3 the spectral weights of the cluster sites at $E_{F}=0$ as functions of $U$. The right panel shows the average double occupancy $d_{\text {occ }}$ $=\sum_{i}\left\langle n_{i \uparrow} n_{i \downarrow}\right\rangle / 3$. Both quantities exhibit hysteresis for increasing and decreasing $U$, indicating coexistence of metallic and insulating solutions.

Finally, Fig. 4 shows the spectral densities at Coulomb energies below and above the Mott transition for $T=0.02 t$ and $t^{\prime}=0.8 t$. Plotted is the average over the three inequivalent sites within the unit cell. Since we are here concerned with the metal-insulator transition we give the ED cluster spectra which can be evaluated without requiring extrapolation from the Matsubara to real frequencies. In the metallic phase the spectra reveal large quasiparticle weight at low frequencies as well as upper and lower Hubbard bands at high frequencies. The insulating phase exhibits a Mott gap as well as pronounced spectral weight in the region of the Hubbard bands. Qualitatively similar features are also seen for the unfrustrated square lattice. ${ }^{34,39}$

\section{CONCLUSION}

In conclusion, the phase diagrams of the Hubbard model for the isotropic and anisotropic triangular lattices have been

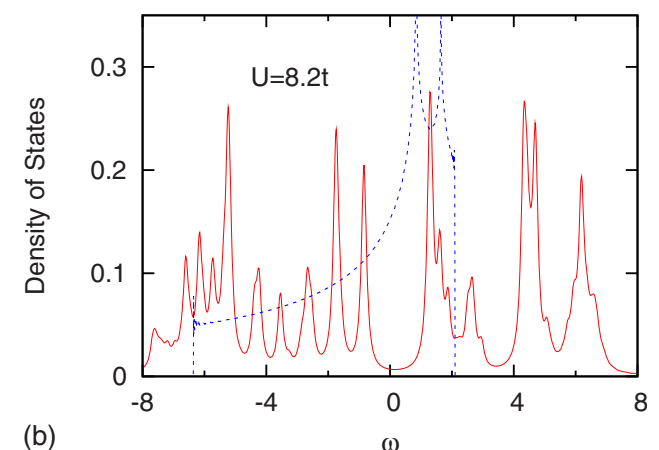

FIG. 4. (Color online) Average spectral distributions of cluster sites below and above the Mott transition for the anisotropic triangular lattice $\left(t=1, t^{\prime}=0.8 t\right.$, and $\left.T=0.02 t\right)$ for Coulomb energies $U=7.5 t$ and $U=8.2 t$. The bare density of states is shown by the dashed blue curve. 
determined within cluster DMFT and exact diagonalization. For moderate frustration, $t^{\prime}=0.8 t$, re-entrant behavior is found and the phase boundaries of the metal-insulator coexistence region are in qualitative agreement with the $T / P$ phase diagram observed experimentally for the anisotropic organic salt $\kappa-\mathrm{Cl}$. The re-entrant behavior disappears in the fully frustrated limit, $t^{\prime}=t$, in agreement with measurements on the nearly isotropic compound $\kappa$-CN. The phase diagram then bears overall resemblance to the one obtained within local DMFT, i.e., in the absence of intersite correlations. The critical temperatures, $T_{c} \approx 50 \mathrm{~K}$ for the isotropic and aniso- tropic lattices, are consistent with the data for $\kappa-\mathrm{CN}$ and $\kappa-\mathrm{Cl}$. These results should also be relevant for the phase diagram of $\left[\mathrm{Pd}(\mathrm{dimt})_{2}\right]_{2}$ salts exhibiting small deviations from the isotropic lattice. ${ }^{40}$

\section{ACKNOWLEDGMENTS}

Computational work (A.L.) was carried out on the Juelich JUMP. J.M. thanks MCI for financial support under Grant No. CTQ2008-06720-C02-02/BQU.
${ }^{1}$ T. Komatsu, N. Matsukawa, T. Inoue, and G. Saito, J. Phys. Soc. Jpn. 65, 1340 (1996).

${ }^{2}$ H. Ito, T. Ishiguro, M. Kubota, and G. Saito, J. Phys. Soc. Jpn. 65, 2987 (1996).

${ }^{3}$ S. Lefebvre, P. Wzietek, S. Brown, C. Bourbonnais, D. Jérome, C. Mézière, M. Fourmigué, and P. Batail, Phys. Rev. Lett. 85, 5420 (2000).

${ }^{4}$ P. Limelette, P. Wzietek, S. Florens, A. Georges, T. A. Costi, C. Pasquier, D. Jérome, C. Mézière, and P. Batail, Phys. Rev. Lett. 91, 016401 (2003).

${ }^{5}$ Y. Shimizu, K. Miyagawa, K. Kanoda, M. Maesato, and G. Saito, Phys. Rev. Lett. 91, 107001 (2003).

${ }^{6}$ F. Kagawa, T. Itou, K. Miyagawa, and K. Kanoda, Phys. Rev. B 69, 064511 (2004).

${ }^{7}$ Y. Kurosaki, Y. Shimizu, K. Miyagawa, K. Kanoda, and G. Saito, Phys. Rev. Lett. 95, 177001 (2005).

${ }^{8}$ S. Ohira, Y. Shimizu, K. Kanoda, and G. Saito, J. Low Temp. Phys. 142, 153 (2006)

${ }^{9}$ M. Imada, A. Fujimori, and Y. Tokura, Rev. Mod. Phys. 70, 1039 (1998).

${ }^{10}$ H. Kino and H. Fukuyama, J. Phys. Soc. Jpn. 65, 2158 (1996).

${ }^{11}$ R. H. McKenzie, Science 278, 820 (1997); Comments Condens. Matter Phys. 18, 309 (1998).

${ }^{12}$ P. W. Anderson, Mater. Res. Bull. 8, 153 (1973).

${ }^{13}$ S. Yamashita, Y. Nakazawa, M. Oguni, Y. Oshima, H. Nojiri, Y. Shimizu, K. Miyagawa, and K. Kanoda, Nat. Phys. 4, 459 (2008).

${ }^{14}$ Y. Shimizu, H. Akimoto, H. Tsujii, A. Tajima, and R. Kato, Phys. Rev. Lett. 99, 256403 (2007).

${ }^{15}$ T. Kashima and M. Imada, J. Phys. Soc. Jpn. 70, 3052 (2001); H. Morita, S. Watanabe, and M. Imada, ibid. 71, 2109 (2002); T. Mizusaki and M. Imada, Phys. Rev. B 74, 014421 (2006).

${ }^{16}$ Y. Imai and N. Kawakami, Phys. Rev. B 65, 233103 (2002).

${ }^{17}$ S. Onoda and M. Imada, Phys. Rev. B 67, 161102 (2003); S. Onoda and N. Nagaosa, J. Phys. Soc. Jpn. 72, 2445 (2003).

${ }^{18}$ O. Parcollet, G. Biroli, and G. Kotliar, Phys. Rev. Lett. 92, 226402 (2004).

${ }^{19}$ S. Watanabe and M. Imada, J. Phys. Soc. Jpn. 73, 3341 (2004).

${ }^{20}$ A. Singh, Phys. Rev. B 71, 214406 (2005).

${ }^{21}$ H. Yokoyama, M. Ogata, and Y. Tanaka, J. Phys. Soc. Jpn. 75,
114706 (2006)

${ }^{22}$ B. Kyung and A.-M. S. Tremblay, Phys. Rev. Lett. 97, 046402 (2006); B. Kyung, Phys. Rev. B 75, 033102 (2007).

${ }^{23}$ P. Sahebsara and D. Senechal, Phys. Rev. Lett. 97, 257004 (2006); 100, 136402 (2008).

${ }^{24}$ T. Koretsune, Y. Motome, and A. Furusaki, J. Phys. Soc. Jpn. 76, 074719 (2007).

${ }^{25}$ T. Senthil, Phys. Rev. B 78, 045109 (2008).

${ }^{26}$ T. Ohashi, T. Momoi, H. Tsunetsugu, and N. Kawakami, Phys. Rev. Lett. 100, 076402 (2008).

${ }^{27}$ H. Lee, G. Li, and H. Monien, Phys. Rev. B 78, 205117 (2008).

${ }^{28}$ D. Galanakis, T. D. Stanescu, and Ph. Phillips, Phys. Rev. B 79, 115116 (2009).

${ }^{29}$ A. Georges, G. Kotliar, W. Krauth, and M. J. Rozenberg, Rev. Mod. Phys. 68, 13 (1996).

${ }^{30}$ M. Caffarel and W. Krauth, Phys. Rev. Lett. 72, 1545 (1994).

${ }^{31}$ A. I. Lichtenstein and M. I. Katsnelson, Phys. Rev. B 62, R9283 (2000); G. Kotliar, S. Y. Savrasov, G. Palsson, and G. Biroli, Phys. Rev. Lett. 87, 186401 (2001).

${ }^{32}$ A. Liebsch, H. Ishida, and J. Merino, Phys. Rev. B 78, 165123 (2008); see also C. A. Perroni, H. Ishida, and A. Liebsch, ibid. 75, 045125 (2007).

${ }^{33}$ W. Y. Ching, Y. N. Xu, Y. C. Jean, and Y. Lou, Phys. Rev. B 55, 2780 (1997).

${ }^{34}$ H. Park, K. Haule, and G. Kotliar, Phys. Rev. Lett. 101, 186403 (2008).

${ }^{35}$ J. Merino, B. J. Powell, and R. H. McKenzie, Phys. Rev. B 73, 235107 (2006).

${ }^{36}$ K. Aryanpour, W. E. Pickett, and R. T. Scalettar, Phys. Rev. B 74, 085117 (2006).

${ }^{37}$ See also M. Vekić and S. R. White, Phys. Rev. B 47, 1160 (1993).

${ }^{38}$ A. E. Trumper, Phys. Rev. B 60, 2987 (1999); J. Merino, R. H. McKenzie, J. B. Marston, and C.-H. Chung, J. Phys.: Condens. Matter 11, 2965 (1999); W. Zheng, R. R. P. Singh, R. H. McKenzie, and R. Coldea, Phys. Rev. B 71, 134422 (2005).

${ }^{39}$ Y. Z. Zhang and M. Imada, Phys. Rev. B 76, 045108 (2007).

${ }^{40}$ R. Kato, A. Tajima, A. Nakao, and M. Tamura, J. Am. Chem. Soc. 128, 10016 (2006). 